\title{
The precision of gingival recession measurements is increased by an automated curvature analysis method
}

\author{
Marko Kuralt ${ }^{1,2^{*}} \mathbb{D}$, Rok Gašperšič ${ }^{3,4}$ and Aleš Fidler ${ }^{1,5}$
}

\begin{abstract}
Background: The extent of gingival recession represents one of the most important measures determining outcome of periodontal plastic surgery. The accurate measurements are, thus, critical for optimal treatment planning and outcome evaluation. Present study aimed to introduce automated curvature-based digital gingival recession measurements, evaluate the agreement and reliability of manual measurements, and identify sources of manual variability.

Methods: Measurement of gingival recessions was performed manually by three examiners and automatically using curvature analysis on representative cross-sections $(n=60)$. Cemento-enamel junction (CEJ) and gingival margin (GM) measurement points selection was the only variable. Agreement and reliability of measurements were analysed using intra- and inter-examiner correlations and Bland-Altman plots. Measurement point selection variability was evaluated with manual point distance deviation from an automatic point. The effect of curvature on manual point selection was evaluated with scatter plots.
\end{abstract}

Results: Bland-Altman plots revealed a high variability of examiner's recession measurements indicated by high $95 \%$ limits of agreement range of approximately $1 \mathrm{~mm}$ and several outliers beyond the limits of agreement. CEJ point selection was the main source of examiner's variability due to smaller curvature values than GM, i.e., median values of $-0.98 \mathrm{~mm}^{-1}$ and $-4.39 \mathrm{~mm}^{-1}$, respectively, indicating straighter profile for CEJ point. Scatter plots revealed inverse relationship between curvature and examiner deviation for CEJ point, indicating a threshold curvature value around $1 \mathrm{~mm}^{-1}$

Conclusions: Automated curvature-based approach increases the precision of recession measurements by reproducible measurement point selection. Proposed approach allows evaluation of teeth with indistinguishable CEJ that could be not be included in the previous studies.

Keywords: Gingival recession, Computer-assisted image processing, Dental models, Cementoenamel junction, Observer variation, Computer-assisted image interpretation reproducibility of results

\section{Background}

The extent of gingival recession, i.e., recession depth, is evaluated before and after treatment and represents one of the most important measures determining treatment

\footnotetext{
*Correspondence: marko.kuralt@kclj.si

${ }^{1}$ Department of Restorative Dentistry and Endodontics, University

Medical Centre Ljubljana, Hrvatski trg 6, 1000 Ljubljana, Slovenia

Full list of author information is available at the end of the article
}

outcome [1]. Recession depth is a measure used to quantify treatment success in terms of percentage of root coverage and defects with complete root coverage, enabling comparison between different treatment techniques [1, 2]. Therefore, the accuracy of recession depth measurements is critical for diagnosis, optimal treatment planning, and outcome evaluation. 
The standard method for gingival recession evaluation is an assessment with the periodontal probe, allowing precise evaluation at a clinically acceptable level [3, 4]. However, the following limitations should be considered, i.e., variations in position and angulation of the periodontal probe and rounding errors [5-7]. Rounding errors were eliminated with utilization of digital callipers with resolution of $0.01 \mathrm{~mm}$ either with pair of dividers [8] or endodontic spreader [9]. While, 3D digital measuring method $[10,11]$ eliminated both limitations. In brief, the measurement site and direction were defined by selecting a cross-section aligned with the tooth's long axis, eliminating the variations in position and angulation of the periodontal probe. At the same time, utilisation of a digital ruler allowing for measurements to the nearest 0.01 $\mathrm{mm}$ increased the measurement's accuracy and eliminated rounding of measurements to the nearest $\mathrm{mm}$ as with a periodontal probe.

By definition, recession depth is defined as a distance between anatomical landmark, i.e., the cemento-enamel junction (CEJ), and gingival margin (GM) [12]. Thus, the accuracy of recession depth measurements strongly depends on the measurement point selection. The precise determination of the CEJ was already outlined as a significant limitation, reducing the reliability and reproducibility of recession depth measurements [13, 14]. Furthermore, precise localisation of CEJ is hampered by poor visibility, carious or non-carious cervical lesions or cervical restorations [15, 16]. Pini-Prato et al. [17] showed that almost $40 \%$ of teeth with gingival recession exhibit unidentifiable CEJ, rendering repeated measurements using the CEJ nearly impossible and prone to errors during follow-up.

By definition, from medical image analysis, landmarks are well-defined points that can be identified based on distinguishable shape features and can be placed manually by examiners or detected automatically $[18,19]$. With recent advancements, automated image analysis based on geometric properties is gaining increased importance in dentistry [20-24]. One of the widely used geometric properties is curvature [25, 26], a mathematical measure describing a deviation of a curve from being a straight line. In dentistry, curvature analysis was already applied for facial profile landmarking in orthodontics [27] and teeth dimension measurements [28], exhibiting reliable and reproducible evaluation. Up to now, the curvature analysis has not been used in the evaluation of gingival recessions, despite wide variety of utilized digital methods [29].

The present study aimed to (1) introduce automated digital measurements of recession depth by using curvature analysis, (2) evaluate the agreement and reliability of manual digital measurements with examiners with different level of experience, (3) identify the sources of examiner's variability by using curvature analysis, and (4) evaluate the agreement of automated digital and clinical measurements.

\section{Methods \\ Sample}

An existing dataset of digital dental models of patients presenting with gingival recessions referred to the Department of Oral Medicine and Periodontology, University Medical Centre Ljubljana, Slovenia, was used to compare digital measurements of gingival recessions. The digital dental models were acquired by intraoral scanning (CEREC Omnicam AC, Dentsply Sirona; software version: SW 4.5.2) when the first author, i.e., experienced operator, was present at the Department in the year 2019. Thus, the dataset is representing a convenience sample. The National Medical Ethics Committee approved the study (Protocol No. 0120-595/2018/4), and the study was conducted in accordance with the Helsinki Declaration as revised in 2013.

The complete dataset was screened for study eligibility by the first author prior to study entry. Digital dental models were eligible for inclusion when gingival recession defect was present at any tooth either in the upper or lower arch and only when the affected teeth presented with identifiable natural CEJ, i.e., Class A- and A + in the Classification of root surface concavities by Pini-Prato et al. [17]. In cases where multiple digital dental models from the same patient were available, e.g., follow-up models after root coverage, only the baseline model was used. In patients presented with multiple gingival recessions, each gingival recession defect was referred to as an individual unit.

The study sample consisted of digital dental models obtained from ten patients, resulting in 52 teeth with gingival recession with identifiable natural CEJ (Additional file 1). Due to the inclusion of molars with the gingival recession at both roots at the buccal side, 60 measuring sites were collected. The distribution of measuring sites per tooth group were: 10 incisors ( 7 maxillary and 3 mandibular), 10 canines (9 maxillary and 1 lower), 20 premolars (18 maxillary and 2 lowers), and 20 molars (all maxillary).

\section{Comparison of digital measurements of recession depth} Included digital dental models were then imported into the 3D data measurement analysis software (GOM Inspect 2017; GOM GmbH). A local coordinate system was created for each tooth of interest, with a long axis of the tooth matching the $z$-axis and mesio-distal direction matching the $x$-axis. Digital dental models were refined with "Refine mesh" tool (with "Minimal edge 
length" setting set at $0.01 \mathrm{~mm}$ ) to enhance the digital model quality and resolution. A representative buccooral cross-section was then manually selected at the central buccal site in the direction of the tooth's long axis. In molars, a bucco-oral cross-section was created for each root if the gingival recession was present at the corresponding root.

Measurements of gingival recessions were performed independently by manual and automatic approach (Additional file 2). In the cross-section images, two measurement points, representing CEJ and GM, were determined manually and automatically, followed by an automated distance measurement.

For the manual method, three examiners with various level of digital image analysis experience performed the measurement point selection on the cross-sections (Fig. 1a-c). Examiner No. 1, general dental practitioner with 9 years of clinical experience, was without previous experience in digital image analysis. Examiner No. 2, endodontist, was experienced in digital analysis with over 20 years of experience. Examiner No. 3, periodontist, was without previous experience in digital image analysis, but was experienced in clinical diagnostics, i.e., a standard calibrated examiner for clinical periodontal measurements. The process of measurement point selection was repeated one week later by all three examiners. The order of cross-sections was randomised for each examiner, reducing the possible bias in the repeated measurements.

For the automatic method, the shape of each crosssection was mathematically described by the curvature. Curvature was computed by the software's "Compute Curve Curvature" tool describing a cross-section curve with a mathematical function, i.e., spline function. From this spline curve, curvature values were determined for each point at a distance of $0.01 \mathrm{~mm}$. Obtained curvature values were then visualised with colour-coded "curvature combs" [30], i.e., graphs that display the amount of curvature at a specific point on the cross-section (Fig. 1d).

Based on the obtained curvature values, two crosssection segments of interest were recognised on all crosssections, i.e., CEJ and GM. CEJ segment is represented by the transition from convex to concave shape in coronoapical direction. In contrast, the GM segment is represented by the transition from convex to concave shape in the opposite direction, i.e., apico-coronal. For precise measurement point selection, local extreme curvature values in the concave part of both segments mentioned above were determined as CEJ and GM points (Fig. 1d). GM point was determined based on the established gingival margin definition [11, 31]. CEJ point decision was
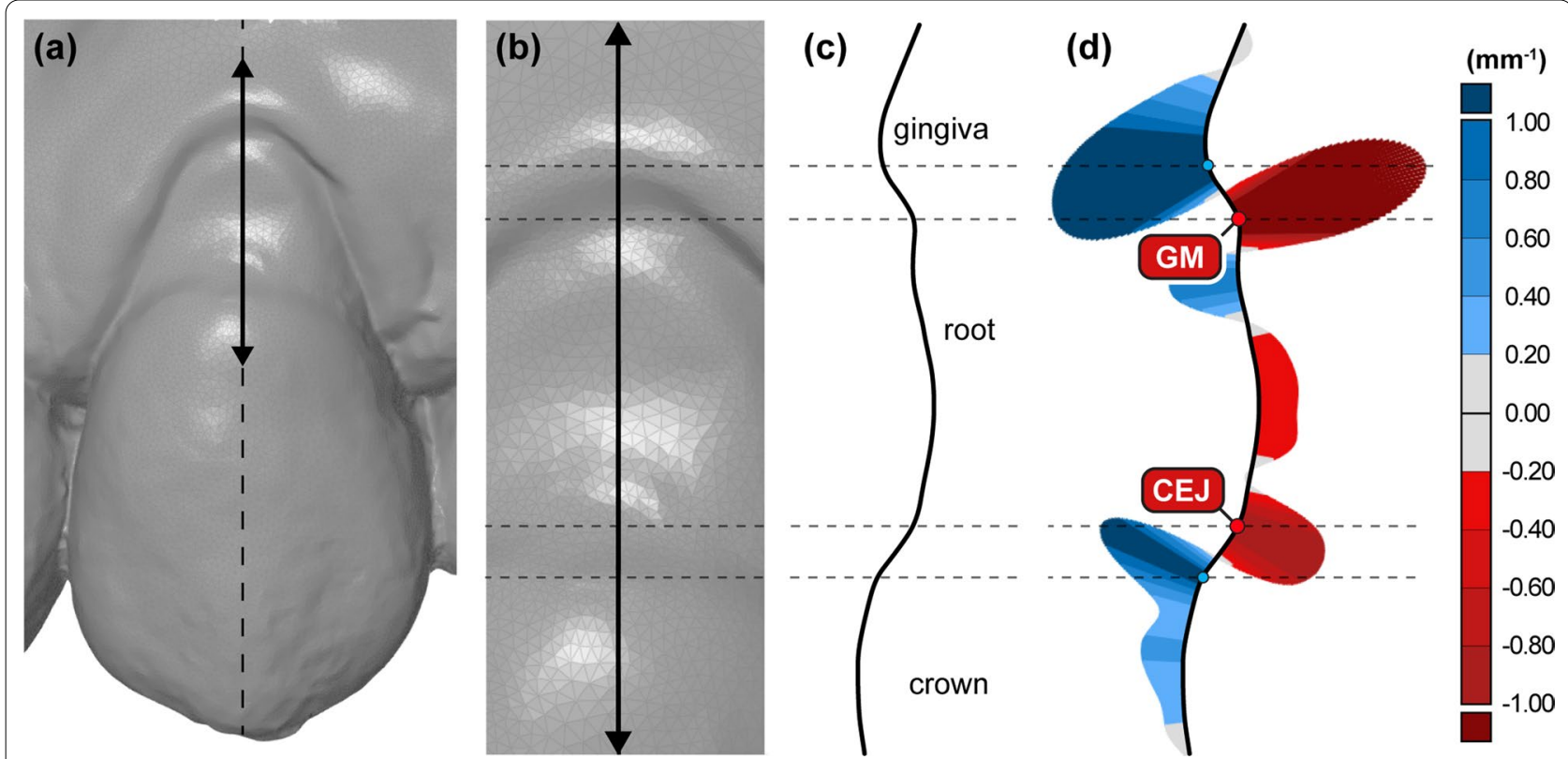

Fig. 1 Automated measurement point selection by using curvature analysis on a representative cross-section. Digital model of maxillary left canine with a representative cross-section at the central buccal site in the direction of the long axis of the tooth (full black line) (a) and a corresponding close-up view (b). A side view of a cross-section (c) also depicting the view for manual measurement point selection. A "curvature comb", i.e., a graph that displays the curvature values at a specific point on an actual cross-section, is shown at (d). Two cross-section segments of interest are marked with dashed grey lines, defined with two local extreme points with positive and negative curvature, i.e., maximum convexity (blue) and concavity (red), respectively. Both cemento-enamel junction (CEJ) and gingival margin (GM) points are defined as a local extreme point with maximum concavity (red) within the cross-section segments mentioned above 
based on existing literature [28, 32-34]. After the determination of points of interest, points are then selected automatically.

After either manual or automatic measurement point selection, a gingival recession was measured automatically as a distance between the measurement points.

\section{Evaluation of manual measurement point selection variability}

Measurement point selection variability was measured as a distance of manual measurement point in reference to automated curvature-based point position on a crosssection. The distance was obtained with the software's "Arc length" tool. It was performed for both the CEJ and GM measurement points. Results were presented using box-plots with positive values representing coronal deviation and negative values apical deviation of manual measurement points.

Furthermore, for CEJ measurement point, the effect of curvature on manual point selection deviation was evaluated by creating curvature vs. examiner deviation scatter plots.

\section{Comparison of digital and clinical recession depth measurements}

Obtained automated and manual digital measurements were compared to clinical measurements of recession depth. Clinical measurements were collected retrospectively from patient's records and were performed by Examiner No. 3.

\section{Statistical analysis}

Statistical analysis was carried out using a statistical software program (IBM SPSS Statistics version 25; IBM Corp). Descriptive statistics, including mean, standard deviations, median, minimum, and maximum values, were obtained.

For evaluation of the examiners' bias, i.e., reliability or inter-examiner variability, and ability of the examiners to repeat multiple measurements, i.e., reproducibility or intra-examiner variability, the intraclass correlation coefficient (ICC) and confidence intervals were calculated.

Agreement of the digital measurements between examiners was analysed using Bland-Altman plots using the curvature-based approach as a reference. The mean differences between tested groups were assessed using the one-sample $\mathrm{t}$-test to determine if mean differences were statistically different from zero.

Agreement of the clinical and digital approach was also analysed using Bland-Altman plot using the clinical approach as a reference and the mean differences between tested groups assessed using the one-sample t-test.

\section{Results}

Recession depth measurements

Median recession depth obtained with an automated curvature-based approach for a study sample $(n=60)$ was $1.90 \mathrm{~mm}$ (25th-75th percentile: $1.25-2.62 \mathrm{~mm}$ ), representing a sample of predominately shallow gingival defects.

\section{Agreement and reliability of digital recession depth measurements}

Based on the inter- and intra-examiner reliability with high lower boundary values of the 95\% confidence intervals, i.e., 0.93 and 0.94 , respectively, the manual approach can be considered excellent regarding precision.

The mean differences (and 95\% confidence interval) between the manual and automated approach on Bland-Altman plots were around zero, i.e., $-0.12 \mathrm{~mm}$ $(-0.18--0.07), 0.04 \mathrm{~mm}(-0.03-0.11)$, and $-0.04 \mathrm{~mm}$ $(-0.08--0.01)$ for Examiner No. 1, 2, and 3 in Round 1, respectively, and $-0.07 \mathrm{~mm}(-0.13--0.02),-0.03 \mathrm{~mm}$ $(-0.10-0.04)$, and $-0.05 \mathrm{~mm}(-0.10-0.01)$ for Examiner No. 1, 2, and 3 in Round 2, respectively (Fig. 2). A statistically significant mean difference after adjustment for multiple comparisons was found only for Examiner No.1 in the first round of measurements compared to the automated measurements $(p<0.001)$ (Fig. 2a). The high variability of recession depth measurements was found for all three examiners in both rounds, indicated by high $95 \%$ limits of agreement range of approximately $1 \mathrm{~mm}$ and outliers beyond the limits of agreement (Fig. 2).

\section{Manual measurement point selection deviation}

A small median difference was found for both CEJ and GM points, except for Examiner No. 1's first-round (Fig. 3). High variability was found for CEJ compared to the GM point, indicated by a high interquartile range and several outliers (Fig. 3a).

The overall shape description of CEJ and GM profiles using curvature showed that CEJ points exhibit lower curvature values than GM points, with median (5th-95th percentile) of $0.99 \mathrm{~mm}^{-1}(0.32-2.30)$ and $4.39 \mathrm{~mm}^{-1}$ (1.98-9.14) for CEJ and GM, respectively. Values and box-plots also revealed that $C E J$ points exhibit a smaller range than GM points (Fig. 4), despite visually more considerable shape differences between CEJ profiles, as depicted by visual inspection of cross-sections (Fig. 5). 


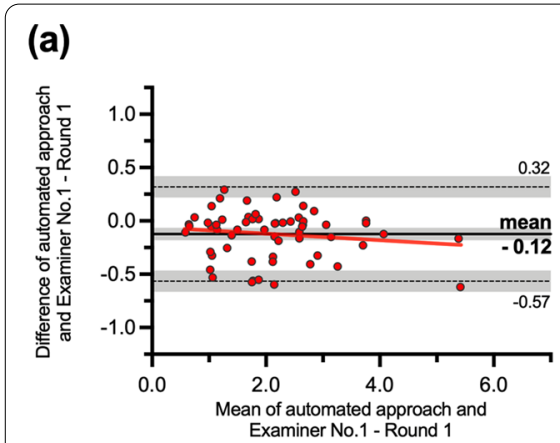

(d)

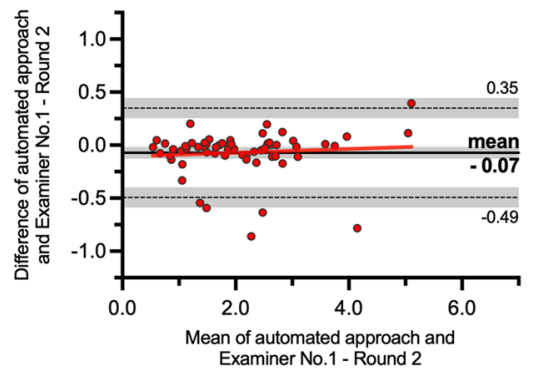

(b)

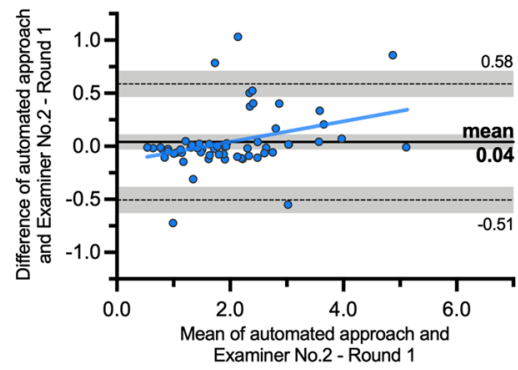

(e)

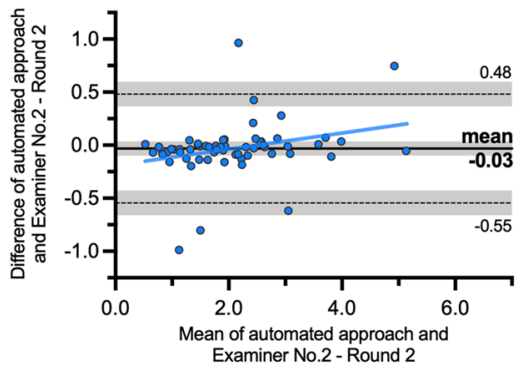

(c)

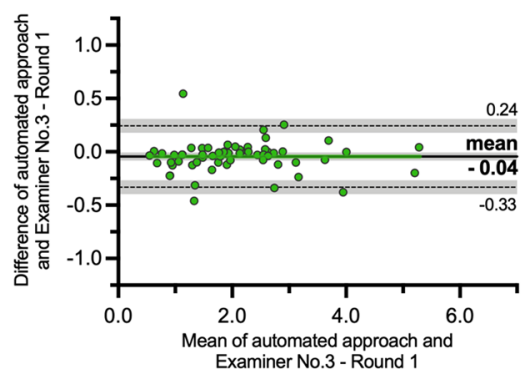

(f)

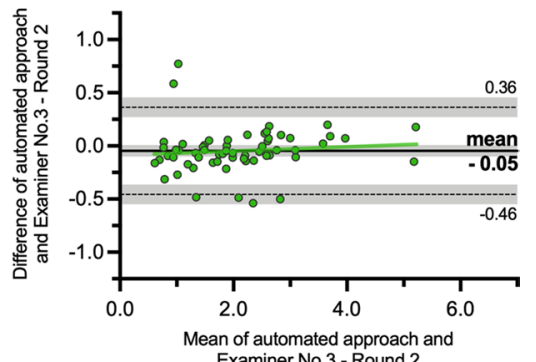

Fig. 2 Bland-Altman plots showing a comparison of digital measurements of gingival recession between Examiner No. 1 (red dots), No. 2 (blue dots), and No. 3 (green dots) in the first (a-c) and second round of measurements ( $\mathbf{d}-\mathbf{f})$ in reference to automated curvature-based approach. The $x$-axis indicates the mean measurement of the gingival recession between compared approaches. The $y$-axis indicates the difference between compared approaches. A black line with the surrounding grey area indicates mean bias and 95\% confidence interval. A dashed black line with a surrounding grey area indicates either upper or lower 95\% limits of agreement and corresponding 95\% confidence interval
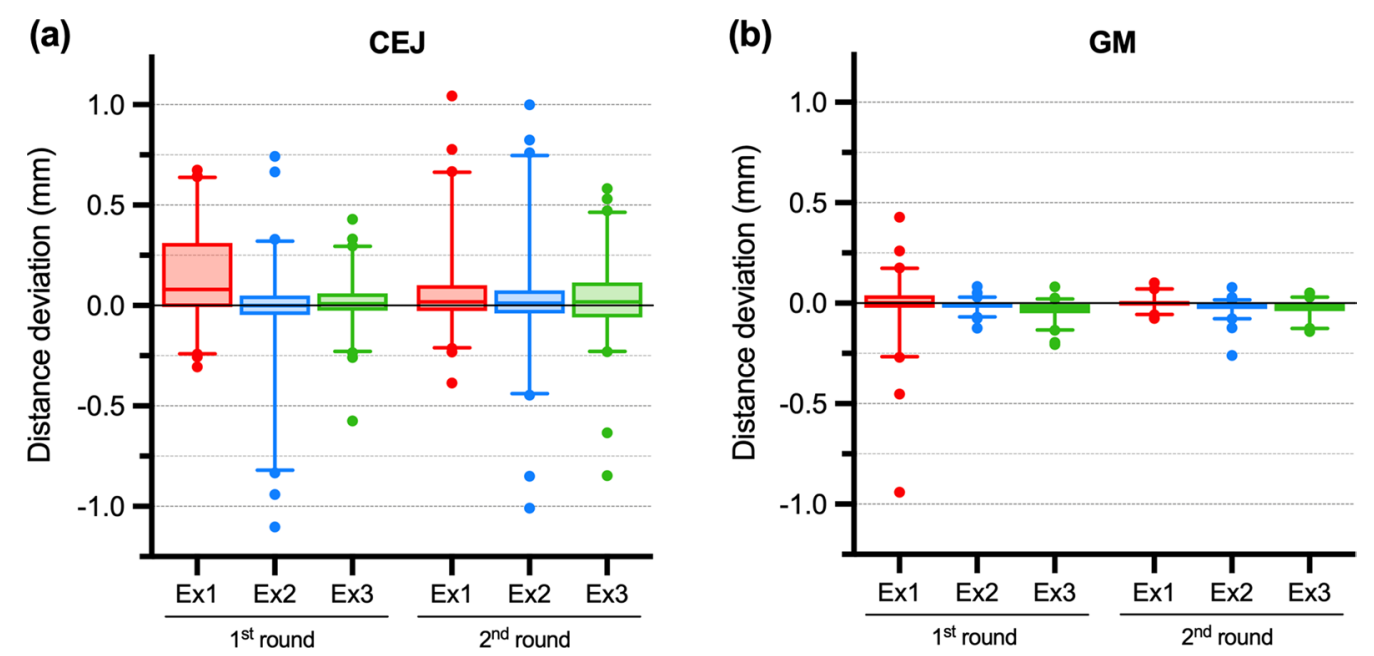

Fig. 3 Manual measurement point selection variability depicted with box-plots with measurement point distance deviation of cemento-enamel junction (CEJ) (a) and gingival margin (GM) point (b) for each examiner and a round of measurement in reference to automated curvature-based point position on a cross-section. Box represent 25th and 75th percentile, while whisker represents 5th and 95th percentile and dots represent outliers

The scatter plots revealed inverse relationship between curvature and examiner deviation distance for CEJ point, indicating a threshold curvature value around $1 \mathrm{~mm}^{-1}$
(Fig. 6), with the exception of Examiner No. 1's first round of measurements (Fig. 6a). Below this threshold value, the variability of deviation increase. 


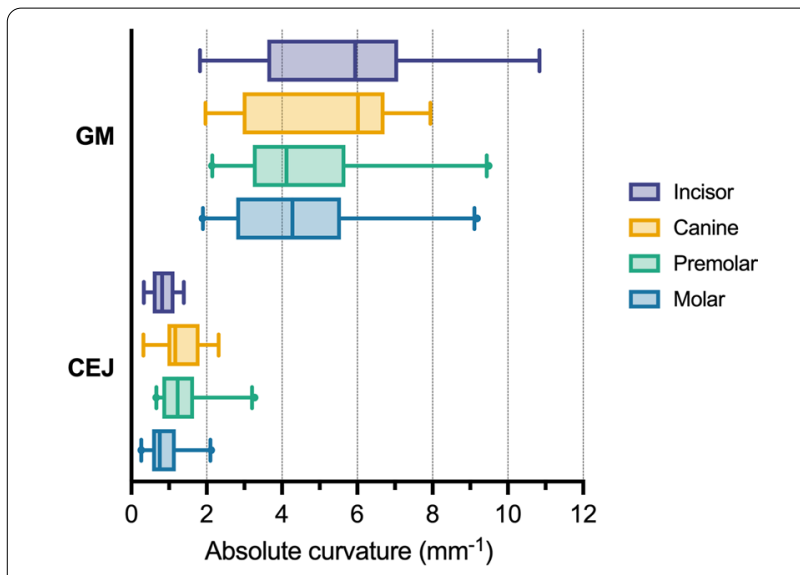

Fig. 4 Profile shape description of cemento-enamel junction (CEJ) and gingival margin (GM) points using box-plots split by tooth group. Box represent the 25 th and 75 th percentile, while whiskers represent the 5 th and 95 th percentile, and dots represent outliers

\section{Agreement of digital and clinical recession depth measurements}

The mean differences (and 95\% confidence interval) between the clinical and manual digital approach on Bland-Altman plots were $0.40 \mathrm{~mm}(0.20-0.59), 0.56 \mathrm{~mm}$ (0.39-0.73), and $0.48 \mathrm{~mm}(0.30-0.66)$ for Examiner No. 1,2 , and 3 in Round 1, respectively, and $0.45 \mathrm{~mm}(0.27-$ $0.62), 0.49 \mathrm{~mm}(0.32-0.66)$, and $0.47 \mathrm{~mm}(0.29-0.66)$ for Examiner No. 1, 2, and 3 in Round 2, respectively
(Additional file 3: Fig. S1). The mean difference (and 95\% confidence interval) between the clinical and automated digital approach was $0.52 \mathrm{~mm}(0.34-0.70)$. A statistically significant mean difference after adjustment for multiple comparisons was found for all comparisons. The high variability of recession depth measurements was found for the clinical approach, indicated by high 95\% limits of agreement range of approximately $2.7 \mathrm{~mm}$ (Additional file 3: Fig. S1).

\section{Discussion}

Accurate evaluation of gingival recession dimensions is an essential part of the diagnosis, treatment planning, and outcome evaluation. In the present study, curvature analysis was used to accurately automate the measurement point selection required for recession depth measurement and analyse the examiners' measurement error. The main source of variability for examiners was CEJ measurement point selection. The automated measurements of recession depth using curvature analysis reduce human variability and increase the precision of measurements.

In the present study, a sample of predominately shallow gingival defects was used to compare approaches due to the inclusion of teeth with identifiable natural CEJ only. Shallow gingival defects presenting with small recession depths are hard to evaluate with a periodontal probe due to mentioned limitations of measurement accuracy [5-7]. However, as already outlined by Zuhr et al. [10],

\section{(a)}

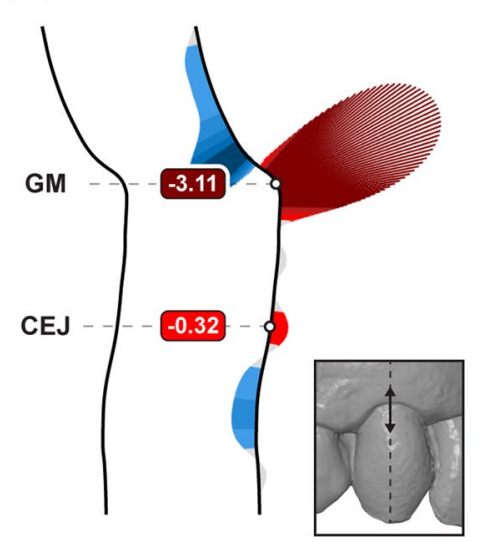

(b)

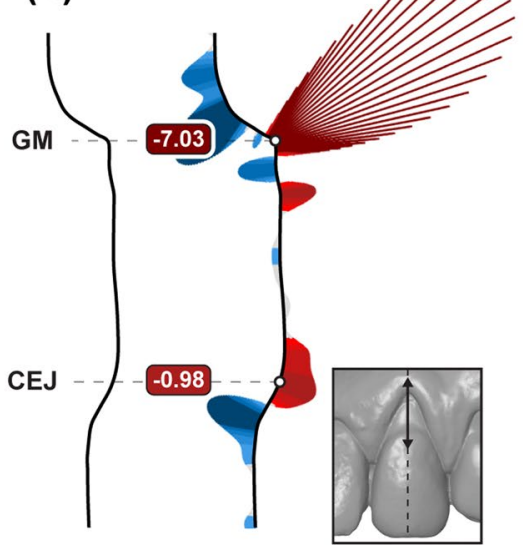

(c)

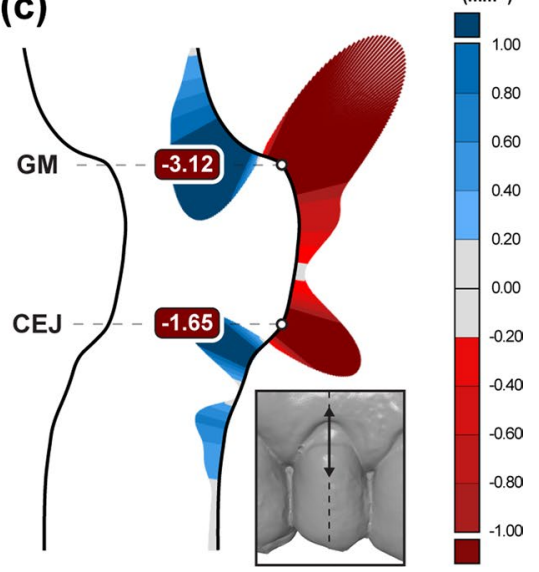

Fig. 5 Cross-section shapes of representative gingival margin (GM) and cemento-enamel junction (CEJ) profiles with "curvature combs" graphs of three maxillary teeth, i.e., right canine (a), left canine (b), and right first premolar (c). Visual inspection of cross-section profiles and "curvature comb" graphs depicts GM point as more distinct and easier to select than CEJ point. Visually perceived shape differences at CEJ points compared to GM points are larger despite the smaller differences in curvature values, i.e., $-0.32 \mathrm{~mm}^{-1}$ vs. $-0.98 \mathrm{~mm}^{-1}$ for CEJ points and $-3.11 \mathrm{~mm}{ }^{-1}$ vs. $-7.03 \mathrm{~mm}^{-1}$ for GM points ( $\mathbf{a}$ and $\mathbf{b}$, respectively). It results from the mathematical definition of curvature as a ratio, i.e., an inverse radius (1/r), and human perception, which more easily distinguish between the straight line and a circle than between two circles with a small difference in radius. Interestingly, similar GM curvature values in a and c produce different shapes of curvature comb graphs (figure c "curvature comb" in coronal direction), which can be explained as an effect of root surface defect present in $\mathbf{c}$ 


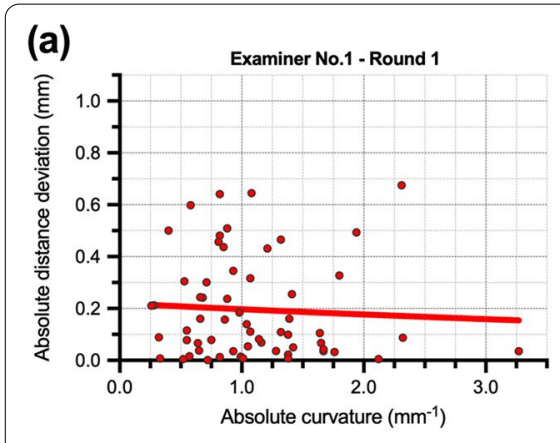

(d)

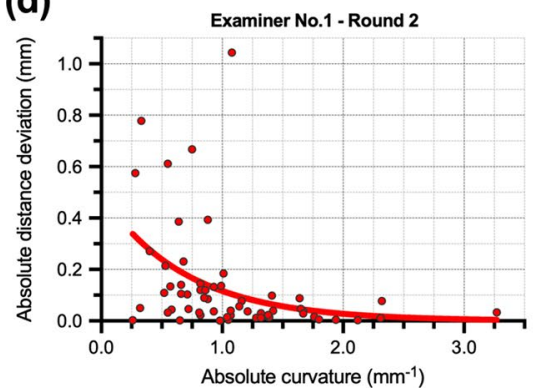

(b)

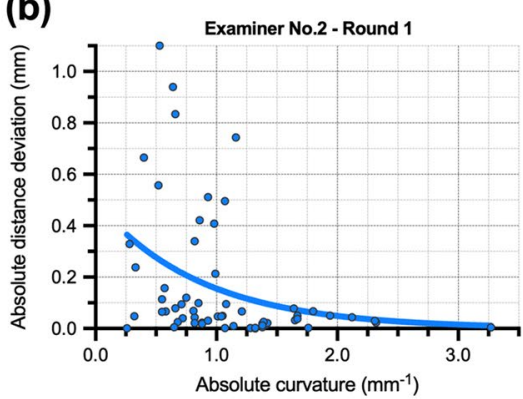

(e)

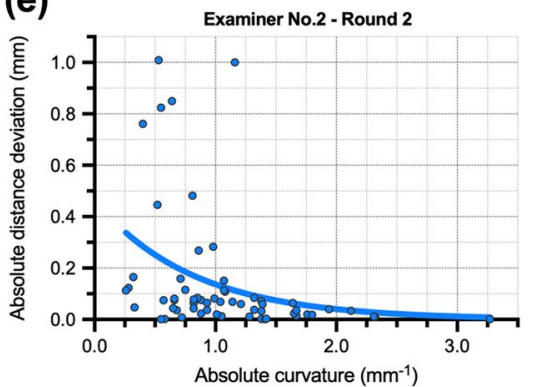

(c)

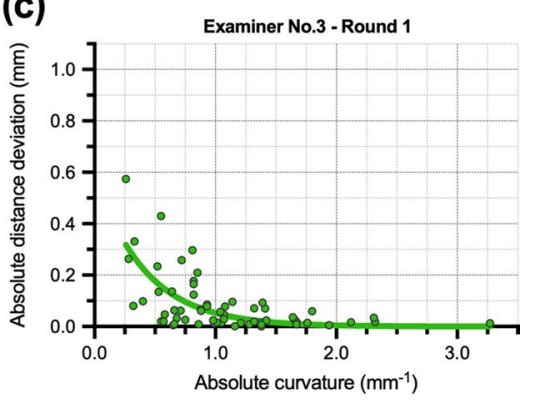

(f)

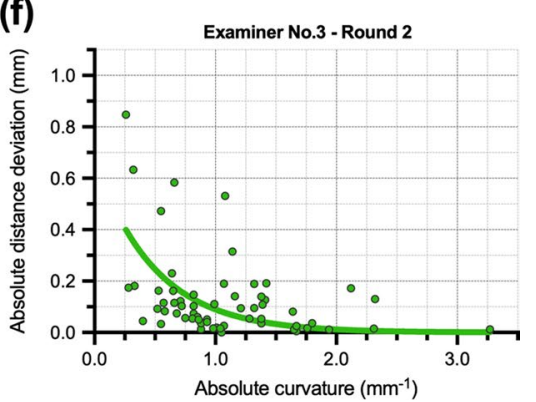

Fig. 6 Scatter plots displaying correlation of curvature and manual CEJ points distance deviation, i.e., the distance between manual and automated curvature-based CEJ reference point position. The upper row shows the first round of measurement (a-c), and the lower row shows the second round of measurements (d-f). The same colour legend is used as in Figs. 2 and 3 and i.e., Examiner No. 1—red, No. 2-blue, and No. 3-green

increased resolution of digital measurements to the nearest $0.01 \mathrm{~mm}$ enables evaluation of shallow gingival defects as well. Additionally, the importance of measurement accuracy was also emphasised with a superb illustration of the effect of rounding digital measurements on two important parameters for evaluating the success of different treatment techniques, i.e., percentage of root coverage [35] and percentage of defects with complete root coverage [10,35].

Manual digital measurements of gingival recession exhibit high variability depicted by Bland-Altman plots. Errors are an inherent part of manual measurements and are unavoidable with human involvement [36]. Therefore, for objective comparison of variability between examiners and the unknown true value of recession depth, an automated curvature-based approach was used as a reference method due to automaticity enabling perfect reproducibility of repeated measurements. Despite excellent ICC values and non-significant mean differences between the approaches, Bland-Altman analysis facilitated the comparison of digital approaches in each individual measurement and allowed for the detailed investigation of the performance of each approach in the sample, revealing a relatively high variability of recession depth measurements with a 95\% limit of agreements range of approximately $1 \mathrm{~mm}$. Despite inexperience in digital analysis, Examiner No. 3, being experienced in clinical diagnostics, exhibited the smallest range of $95 \%$ limits of agreement, i.e., $0.57 \mathrm{~mm}$; however, no significant differences can be observed between examiners. Obtained variability range is much smaller than evaluation with a periodontal probe, i.e., around $2.5 \mathrm{~mm}$, and similar to evaluation with a digital manual approach, i.e., around 1 $\mathrm{mm}$, supported by our results (Additional file 2: Fig. S1) and also found in other studies $[3,4]$. In contrast to previous digital studies, the only variable part in the present study was measurement point selection; thus, obtained variability can be attributed solely to the measurement points selection. The digital manual approach utilised in the previous studies $[3,4]$ measured gingival recessions on the digital models and not on cross-sections, including measuring direction and angle variability, into the comparison. The implementation of the proposed approach is straightforward. It requires only a single 3D data measurement analysis software that is free for noncommercial use.

Importantly, the main source of variability is the CEJ measurement point. Both the manual and the automated approach used the shape of the cross-section for the measurement point selection. The main difference between the approaches was the examiners' subjective 
bias. Our results revealed that the main variability could be attributed to the position of the CEJ measurement point (Fig. 3a), despite inclusion criteria with visible and identifiable CEJ. Curvature analysis revealed a straighter CEJ profile compared to the GM profile, outlined with smaller absolute curvature values (Fig. 4), resulting in a less distinct shape feature for CEJ than the GM (Fig. 5), despite different tooth groups. Our findings were supported by scatter plots revealing that absolute curvature values of approximately $1 \mathrm{~mm}^{-1}$ represent an arbitrary threshold where higher deviations in measurement point position can be observed (Fig. 6). To outline the magnitude of the problem, half of the cases exhibit CEJ with curvature less than $1 \mathrm{~mm}^{-1}$. Furthermore, a great example is comparing the first and second round of measurements for Examiner No. 1 with larger deviations present at higher absolute curvature values as well in the first round. In contrast, in the second round, a "learning effect" was observed with improving precision over the higher range, but importantly, not below the mentioned threshold.

This study was subject to some limitations. First, either measurement point's true position is impossible to determine; therefore, the trueness of measurements could not be evaluated. Despite the perfect reproducibility of point selection presented automated approach requires initial human input to determine the points of interest. In the present study, the point definition was based on definitions from existing literature [11, 28, 31-34]. However, with time, both the cement and enamel wears away due to the exposure of tooth's root surface to the oral environment [17], rendering CEJ a questionable landmark. The automated curvature-based approach is applicable beyond the limitations of identifiable CEJ, meaning that when defects or restorations are present, the edges of the defect or restorations can be objectively defined by curvature analysis as well. Thus, in digital analysis, the term and landmark of CEJ might have been redefined to "coronal reference point" in the scope of the periodontal measurements. Additionally, curvature analysis can also aid in describing root surface defects' morphology, opening novel insights into the reconstruction of anatomical CEJ before root coverage [37, 38]. However, further research is required for validation in cases without identifiable natural CEJ.

Second, compared to a whole 3D model, a cross-section represents only one out of many available measuring sites. Further research is required to explore novel possibilities analysing whole 3D models. Nonetheless, cross-sections are widely used to evaluate tissue dynamics in periodontal plastic surgery [35, 39-43] and implantology [44-46], enabling great standardisation regarding the selection of measuring site and direction of measurement. Measurement site selection, e.g., selecting a representative cross-section, is also one of the possible variabilities in the measurement of recession depth. For follow-up measurements measuring site variability was eliminated with superimposition of digital models [11, 40], also stating the importance of superimposition accuracy [47].

Third, while the clinical approach utilizes also the colour properties as the additional visual reference, only shape properties were used in the present study. Despite color acquisition of digital dental models in the study, export of color models for further analysis was unavailable due to software limitations. Exporting of color models has become available only recently for some systems and color models proved to be useful in digital measurements of keratinized tissue width with main emphasis on color difference [48]. Further research could be aimed to test the suitability of colour properties on automated CEJ detection.

In periodontology, the potential of digital measurements remains unexploited due to the unclear definitions and inconsistent implementations, inherited from the conventional methods [29]. In treatment planning, the automated digital method would allow for the early detection of initial, i.e., prodromal, recessions due to higher resolution and precision, empowering the appropriate preventive measures. Importantly, the same level of resolution and precision will be maintained through the follow-up period. Such precision through all phases of evaluation will greatly improve the quality as well as the credibility of clinical and research data [29].

\section{Conclusions}

Novel automated approach increases the precision of gingival recession measurements by using curvature analysis, opening novel possibilities for comparison of different treatment techniques. The utilisation of curvature analysis enables completely reproducible measurement point selection, eliminating human variability and seems promising for the evaluation of teeth with indistinguishable CEJ that were often excluded in the previous studies. Therefore, future studies could be done on larger, more clinically relevant samples.

\section{Abbreviations}

3D: Three-dimensional; CEJ: Cemento-enamel junction; GM: Gingival margin.; ICC: Intraclass correlation coefficient.

\section{Supplementary Information}

The online version contains supplementary material available at https://doi. org/10.1186/s12903-021-01858-9. 


\begin{abstract}
Additional file 1. Digital models used in the present study in a STL file format.

Additional file 2. Raw measurements and data used in the present study.

Additional file 3: Figure S1. Bland-Altman plots showing a comparison of clinical and digital manual measurements of gingival recession between Examiner No. 1 (red dots), No. 2 (blue dots), and No. 3 (green dots) in the first ( $a, c$, and e) and second round of measurements (b, $d$, and f) in reference to clinical approach. A comparison of clinical and digital automated measurements in reference to clinical approach (g). The $x$-axis indicates the mean measurement of the gingival recession between compared approaches. The $y$-axis indicates the difference between compared approaches. A black line with the surrounding grey area indicates mean bias and 95\% confidence interval. A dashed black line with a surrounding grey area indicates either upper or lower $95 \%$ limits of agreement and corresponding 95\% confidence interval.
\end{abstract}

\section{Acknowledgements}

The authors thank research assistant Hojka Kuralt, DMD for performing the digital measurements.

\section{Authors' contributions}

MK contributed to conception and design of the study and article, acquired the digital model dataset, performed the analysis, interpret the data, drafted the article, and prepared figures; RG acquired the clinical measurements dataset, performed the analysis, interpret the data, and revised the article; AF contributed to conception and design of the study and article, performed the analysis, interpret the data, and revised the article. All authors have read and approved the manuscript.

\section{Funding}

The study was supported by the Ministry of Higher Education, Science, and Technology, Republic of Slovenia, under grant number P3-0293. The funding body did not involve in the study design, data collection, data analysis, interpretation of data and writing the manuscript.

\section{Availability of data and materials}

Digital models used in the present study are available in this article's Additional file 1. Please cite this article when using the digital models. Additionally, the data supporting the study's findings, i.e., raw measurements and data, are available in Additional file 2. Bland-Altman plots evaluating agreement of digital and clinical recession depth measurements are available as Additional file 3.

\section{Declarations}

\section{Ethics approval and consent to participate}

The National Medical Ethics Committee approved the study (Protocol No. 0120-595/2018/4), and the study was conducted in accordance with the Helsinki Declaration as revised in 2013.

\section{Consent for publication}

Not applicable.

\section{Competing interests}

The authors declare that they have no competing interests.

\section{Author details}

${ }^{1}$ Department of Restorative Dentistry and Endodontics, University Medical Centre Ljubljana, Hrvatski trg 6, 1000 Ljubljana, Slovenia. ${ }^{2}$ Faculty of Medicine, University of Ljubljana, Ljubljana, Slovenia. ${ }^{3}$ Department of Oral Medicine and Periodontology, University Medical Centre Ljubljana, Ljubljana, Slovenia. ${ }^{4}$ Department of Oral Medicine and Periodontology, Faculty of Medicine, University of Ljubljana, Ljubljana, Slovenia. ${ }^{5}$ Department of Endodontics and Operative Dentistry, Faculty of Medicine, University of Ljubljana, Ljubljana, Slovenia.

Received: 23 July 2021 Accepted: 27 September 2021 Published online: 07 October 2021

\section{References}

1. Cortellini P, Bissada NF. Mucogingival conditions in the natural dentition: narrative review, case definitions, and diagnostic considerations. J Clin Periodontol. 2018;45:S190-8. https://doi.org/10.1111/jcpe.12948.

2. Pini-Prato G, Magnani C, Zaheer F, Buti J, Rotundo R. Critical evaluation of complete root coverage as a successful endpoint of treatment for gingival recessions. Int J Periodontics Restor Dent. 2017. https://doi.org/ 10.11607/prd.2400.

3. Schneider D, Ender A, Truninger T, Leutert C, Sahrmann P, Roos M, Schmidlin P. Comparison between clinical and digital soft tissue measurements. J Esthet Restor Dent. 2014;26:191-9. https://doi.org/10.1111/jerd. 12084.

4. Fageeh HN, Meshni AA, Jamal HA, Preethanath RS, Helboub E. The accuracy and reliability of digital measurements of gingival recession versus conventional methods. BMC Oral Health. 2019;19:1-8. https://doi.org/10. 1186/s12903-019-0851-0.

5. Hefti AF. Periodontal probing. Crit Rev Oral Biol Med. 1997;8:336-56. https://doi.org/10.1177/10454411970080030601.

6. Grossi SG, Dunford RG, Ho A, Koch G, Machtei EE, Genco RJ. Sources of error for periodontal probing measurements. J Periodontal Res. 1996;31:330-6. https://doi.org/10.1111/j.1600-0765.1996.tb00500.x.

7. Watts TLP. Probing site configuration in patients with untreated periodontitis: a study of horizontal positional error. J Clin Periodontol. 1989. https:// doi.org/10.1111/j.1600-051X.1989.tb02331.x.

8. Santamaria MP, Suaid FF, Casati MZ, Nociti FH, Sallum AW, Sallum EA. Coronally positioned flap plus resin-modified glass ionomer restoration for the treatment of gingival recession associated with non-carious cervical lesions: a randomized controlled clinical trial. J Periodontol. 2008;79:621-8. https://doi.org/10.1902/jop.2008.070285.

9. Bittencourt S, Del Peloso Ribeiro É, Sallum EA, Sallum AW, Nociti FH, Casati MZ. Comparative 6-month clinical study of a semilunar coronally positioned flap and subepithelial connective tissue graft for the treatment of gingival recession. J Periodontol. 2006;77:174-81. https://doi. org/10.1902/jop.2006.050114.

10. Zuhr O, Rebele SF, Schneider D, Jung RE, Hürzeler MB. Tunnel technique with connective tissue graft versus coronally advanced flap with enamel matrix derivative for root coverage: a RCT using 3D digital measuring methods. Part I. Clinical and patient-centred outcomes. J Clin Periodontol. 2014;41:582-92. https://doi.org/10.1111/jcpe.12178.

11. Rebele SF, Zuhr O, Schneider D, Jung RE, Hürzeler MB. Tunnel technique with connective tissue graft versus coronally advanced flap with enamel matrix derivative for root coverage: a RCT using 3D digital measuring methods. Part II. Volumetric studies on healing dynamics and gingival dimensions. J Clin Periodontol. 2014;41:593-603. https://doi.org/10.1111/ jcpe.12254.

12. Pini Prato, G. Mucogingival deformities. Ann Periodontol. 1999. https:// doi.org/10.1902/annals.1999.4.1.98.

13. Zucchelli G, Testori T, De Sanctis M. Clinical and anatomical factors limiting treatment outcomes of gingival recession: a new method to predetermine the line of root coverage. J Periodontol. 2006;77:714-21. https://doi.org/10.1902/jop.2006.050038.

14. Hug HU, Van 't Hof MA, Spanauf AJ, Renggli HH. Validity of clinical assessments related to the cemento-enamel junction. J Dent Res. 1983;62:8259. https://doi.org/10.1177/00220345830620071301.

15. Heasman PA, Ritchie M, Asuni A, Gavillet E, Simonsen JL, Nyvad B. Gingival recession and root caries in the ageing population: a critical evaluation of treatments. J Clin Periodontol. 2017. https://doi.org/10.1111/jcpe.12676.

16. Heasman PA, Holliday R, Bryant A, Preshaw PM. Evidence for the occurrence of gingival recession and non-carious cervical lesions as a consequence of traumatic toothbrushing. J Clin Periodontol. 2015. https://doi. org/10.1111/jcpe.12330.

17. Pini-Prato G, Franceschi D, Cairo F, Nieri M, Rotundo R. Classification of dental surface defects in areas of gingival recession. J Periodontol. 2010;81:885-90. https://doi.org/10.1902/jop.2010.090631.

18. Ibragimov, B., Vrtovec, T. Landmark-based statistical shape representations, 1st ed., Elsevier Ltd, 2017. https://doi.org/10.1016/B978-0-12810493-4.00005-5.

19. Kim H, Shim E, Park J, Kim YJ, Lee U, Kim Y. Web-based fully automated cephalometric analysis by deep learning, Comput Methods Programs Biomed. 2020. https://doi.org/10.1016/j.cmpb.2020.105513. 
20. Joda T, Bornstein MM, Jung RE, Ferrari M, Waltimo T, Zitzmann NU. Recent trends and future direction of dental research in the digital era. Int J Environ Res Public Health. 2020. https://doi.org/10.3390/ijerph17061987.

21. Wang CW, Huang CT, Hsieh MC, Li CH, Chang SW, Li WC, Vandaele R, Marée R, Jodogne S, Geurts P, Chen C, Zheng G, Chu C, Mirzaalian H, Hamarneh G, Vrtovec T, Ibragimov B. Evaluation and comparison of anatomical landmark detection methods for cephalometric X-ray images: a grand challenge. IEEE Trans Med Imaging. 2015. https://doi.org/10.1109/ TMl.2015.2412951.

22. Wang CW, Huang CT, Lee JH, Li CH, Chang SW, Siao MJ, Lai TM, Ibragimov B, Vrtovec T, Ronneberger O, Fischer P, Cootes TF, Lindner C. A benchmark for comparison of dental radiography analysis algorithms. Med Image Anal. 2016. https://doi.org/10.1016/.media.2016.02.004.

23. Lin PL, Huang PY, Huang PW. Automatic methods for alveolar bone loss degree measurement in periodontitis periapical radiographs. Comput Methods Programs Biomed. 2017;148:1-11. https://doi.org/10.1016/j. cmpb.2017.06.012.

24. Abdolali F, Zoroofi RA, Otake Y, Sato Y. Automated classification of maxillofacial cysts in cone beam CT images using contourlet transformation and spherical harmonics. Comput Methods Programs Biomed. 2017;139:197207. https://doi.org/10.1016/j.cmpb.2016.10.024.

25. Vrtovec T, Likar B, Pernuš F. Quantitative analysis of spinal curvature in 3D: application to CT images of normal spine. Phys Med Biol. 2008. https:// doi.org/10.1088/0031-9155/53/7/006.

26. Keeling $\mathrm{A}, \mathrm{Wu}$ J, Ferrari M. Confounding factors affecting the marginal quality of an intra-oral scan. J Dent. 2017;59:33-40. https://doi.org/10. 1016/j.jdent.2017.02.003.

27. Lippold C, Liu X, Wangdo K, Drerup B, Schreiber K, Kirschneck C, Moiseenko T, Danesh G. Facial landmark localization by curvature maps and profile analysis. Head Face Med. 2014. https://doi.org/10.1186/ 1746-160X-10-54

28. Di Angelo L, Di Stefano P, Bernardi S, Continenza MA. A new computational method for automatic dental measurement: the case of maxillary central incisor. Comput Biol Med. 2016;70:202-9. https://doi.org/10. 1016/j.compbiomed.2016.01.018.

29. Kuralt M, Gašperšič R, Fidler A. Methods and parameters for digital evaluation of gingival recession: a critical review. J Dent. https://doi.org/10. 1016/j.jdent.2021.103793.

30. Farin G. Curvature combs and curvature plots. CAD Comput Aided Des. 2016. https://doi.org/10.1016/j.cad.2016.08.003.

31. Lindhe J, Karring T, Araújo M. Anatomy of Periodontal Tissues. In: Lang NP, Lindhe J, editors. Clin. Periodontol. Implant Dent. New York: John Wiley \& Sons Inc; 2015. p. 4-47.

32. Preshaw PM, Kupp L, Hefti AF, Mariotti A. Measurement of clinical attachment levels using a constant-force periodontal probe modified to detect the Cemento-Enamel junction. J Clin Periodontol. 1999. https://doi.org/ 10.1034/j.1600-051X.1999.260704.X.

33. Jeffcoat MK, Jeffcoat RL, Jens SC, Captain K. A new periodontal probe with automated cemento-enamel junction detection. J Clin Periodontol. 1986. https://doi.org/10.1111/j.1600-051X.1986.tb02222.x.

34. Vandana K, Haneet R. Cementoenamel junction: an insight. J Indian Soc Periodontol. 2014;18:549-54. https://doi.org/10.4103/0972-124X.142437.

35. Zuhr O, Rebele SF, Vach K, Petsos H, Hürzeler MB. Tunnel technique with connective tissue graft versus coronally advanced flap with enamel matrix derivate for root coverage: 2-year results of an RCT using 3D digital measuring for volumetric comparison of gingival dimensions. J Clin Periodontol. 2020. https://doi.org/10.1111/jcpe.13328.

36. Bartlett JW, Frost C. Reliability, repeatability and reproducibility: analysis of measurement errors in continuous variables. Ultrasound Obstet Gynecol. 2008;31:466-75. https://doi.org/10.1002/uog.5256.
37. Cairo F, Pini-Prato GP. A technique to identify and reconstruct the cementoenamel junction level using combined periodontal and restorative treatment of gingival recession. A prospective clinical study. Int J Periodontics Restor Dent. 2010;30:573-81. https://doi.org/10.11607/prd.00. 0945.

38. Zucchelli G, Gori G, Mele M, Stefanini M, Mazzotti C, Marzadori M, Montebugnoli L, De Sanctis M. Non-carious cervical lesions associated with gingival recessions: a decision-making process. J Periodontol. 2011;82:1713-24. https://doi.org/10.1902/jop.2011.110080.

39. Bienz SP, Sailer I, Sanz-Martín I, Jung RE, Hämmerle CHF, Thoma DS. Volumetric changes at pontic sites with or without soft tissue grafting: a controlled clinical study with a 10-year follow-up. J Clin Periodontol. 2017:44:178-84. https://doi.org/10.1111/jcpe.12651.

40. Fons-Badal C, Alonso Pérez-Barquero J, Martínez-Martínez N, JFau-López J, Fons-Font A, Agustín-Panadero R. A novel, fully digital approach to quantifying volume gain after soft tissue graft surgery. A pilot study. J Clin Periodontol. 2019. https://doi.org/10.1111/jcpe.13235.

41. Parvini P, Galarraga-Vinueza ME, Obreja K, de Magini RS, Sader R, Schwarz F. Prospective study assessing three-dimensional changes of mucosal healing following soft tissue augmentation using free gingival grafts. J Periodontol. 2021;92:400-8. https://doi.org/10.1002/JPER.19-0640.

42. M. Kuralt, R. Gašperšič, A. Fidler, 3D computer-aided treatment planning in periodontology: A novel approach for evaluation and visualization of soft tissue thickness, J. Esthet. Restor. Dent. 32 (2020) 457-462. https:// doi.org/10.1111/jerd.12614.

43. Kloukos D, Koukos G, Gkantidis N, Sculean A, Katsaros C, Stavropoulos A. Transgingival probing: A clinical gold standard for assessing gingival thickness. Quintessence Int (Berl). 2021;52:394-401. https://doi.org/10. 3290/j.qi.b937015.

44. Galarraga-Vinueza ME, Obreja K, Magini R, Sculean A, Sader R, Schwarz F. Volumetric assessment of tissue changes following combined surgical therapy of peri-implantitis: a pilot study. J Clin Periodontol. 2020;47:115968. https://doi.org/10.1111/jcpe.13335.

45. Thoma DS, Gasser TJW, Jung RE, Hämmerle CHF. Randomized controlled clinical trial comparing implant sites augmented with a volume-stable collagen matrix or an autogenous connective tissue graft: 3-year data after insertion of reconstructions. J Clin Periodontol. 2020;47:630-9. https://doi.org/10.1111/jcpe.13271.

46. Zeltner M, Jung RE, Hämmerle CHF, Hüsler J, Thoma DS. Randomized controlled clinical study comparing a volume-stable collagen matrix to autogenous connective tissue grafts for soft tissue augmentation at implant sites: linear volumetric soft tissue changes up to 3 months. J Clin Periodontol. 2017:44:446-53. https://doi.org/10.1111/jcpe.12697.

47. Kuralt M, Fidler A. Assessment of reference areas for superimposition of serial 3D models of patients with advanced periodontitis for volumetric soft tissue evaluation. J Clin Periodontol. 2021. https://doi.org/10.1111/ jcpe.13445.

48. Lee JS, Jeon YS, Strauss FJ, Jung HI, Gruber R. Digital scanning is more accurate than using a periodontal probe to measure the keratinized tissue width. Sci Rep. 2020;10:0-8. https://doi.org/10.1038/ s41598-020-60291-0.

\section{Publisher's Note}

Springer Nature remains neutral with regard to jurisdictional claims in published maps and institutional affiliations. 